\title{
Landmarking and strong Allee thresholds
}

\author{
Kim Cuddington $\cdot$ Z. Tasker Hull • \\ Warren J. S. Currie • Marten A. Koops
}

Received: 15 October 2014 / Accepted: 12 January 2015 / Published online: 11 March 2015

(C) The Author(s) 2015. This article is published with open access at Springerlink.com

\begin{abstract}
Mate-finding difficulties in small populations are often postulated to create strong demographic Allee effects that increase the probability of extinction of native species or, similarly, decrease the probability that nonnative species will successfully invade. Many species make use of a restricted number of mating locations, detectable from long-distance, that are not selected for habitat reasons (e.g., hilltopping in butterflies). This 'landmarking' strategy may specifically address the problem of overcoming matefinding difficulties. Using a variant of the birthday problem, we demonstrate that populations which locate a restricted number of mate-finding sites using landmark features may have high probability of successful mating even at very low population densities. Therefore, a strong Allee threshold, if it exists, may be very small, and non-native species that make use of this strategy may have a very good chance of population establishment at low density.
\end{abstract}

Keywords Hilltopping · Birthday problem · Endangered species - Invasive species - Mate encounter .

Establishment $\cdot$ Population growth rate

\section{Introduction}

When the number of non-native individuals introduced to a region is small, a demographic Allee effect may act to

K. Cuddington $(\bowtie) \cdot$ Z. T. Hull

Department of Biology, University of Waterloo,

Waterloo, Ontario, Canada

e-mail: kcudding@uwaterloo.ca

W. J. S. Currie · M. A. Koops

Great Lakes Laboratory for Fisheries and Aquatic Sciences,

Fisheries and Oceans Canada, Burlington, Ontario, Canada reduce invasion success (e.g., Drake and Lodge 2006). The existence of a strong Allee effect, where there is a threshold population density below which population growth is negative (e.g., Deredec and Courchamp 2007), may doom small populations to extinction. Mate-finding difficulties have been identified as the most common component of Allee effect that causes a strong demographic Allee effect (Gascoigne et al. 2009; Kramer et al. 2009). Moreover, it has been predicted that difficulty in mate-finding at low densities can be a major stumbling block for the establishment success of non-native species (Veit and Lewis 1996; Taylor and Hastings 2005; Deredec and Courchamp 2007; Kramer et al. 2008).

While we are accustomed to thinking about mate-finding difficulties as potential controls on the success of invasive species, some mechanisms can lead to success even at a very low density of newly introduced individuals. We examine the impact of one little-studied mechanism of mate-finding, landmarking, on strong Allee thresholds. Gascoigne et al. (2009) point out that there are two main categories of adaptations to mitigate mate-finding Allee effects: mechanisms that increase the efficiency of mate-finding at low density such as calls or chemical signalling (e.g., bioluminescent signalling of deep sea fishes: (Ruxton and Bailey 2005), and mechanisms that reduce the likelihood of low density such as mass spawning (e.g., spawning aggregations of cod: Skjraasen et al. 2011). While mass aggregation may be disrupted at very low density (Reed and Dobson 1993; Rowe and Hutchings 2003), some mate-finding strategies such as pheromone signalling can be quite effective at small population size.

Mate-finding strategies which rely on landscape cues may also be effective at low population density. Rather than being attracted by signalling cues from the opposite sex, individuals may simply move towards particular locations 
in the landscape which can either be detected from long distances (landmarks such as treetops and hilltops: Thornhill and Alcock 1983), or which have previously known coordinates (e.g., site fidelity to mating grounds of the albatross: Dubois et al. 1998). For example, it is estimated that over 1000 insect species find mates at the prominent conical hilltop Mount Morris, Queensland, which rises $350 \mathrm{~m}$ above the surrounding area (Skevington 2008). Of these two versions of landscape-determined mate-finding strategies, the use of landmarks, rather than previous knowledge of mating grounds, will be more robust to low densities, since loss of population density can be associated with a loss of the knowledge or individual use of specific mating grounds (Frank and Brickman 2000). Certainly, the use of prominent landmarks is most relevant to the spread of invasive species, since newly introduced individuals will have no previous knowledge of the landscape.

When population density is low, we may guess that if there are only a few landmarked mate-finding sites over a large area, it is quite unlikely that a male and female may end up at the same location at the same time. For example, there is currently concern that Asian carps of the genus Hypophthalmichthys (Bighead and Silver Carps) may colonize the Great Lakes system (Cudmore et al. 2012). These types of Asian carps require rivers of sufficient size and flow for successful spawning (Costa-Pierce 1992; Kolar et al. 2005), but there are relatively few such rivers on each of the Great Lakes. Kocovsky et al. (2012) identify three rivers connected to Lake Erie as good spawning habitat, and suggest a further three may also have potential. The fact that so few suitable rivers have been identified has led some to suggest that it is unlikely that successful reproduction could take place at low density.

Here, we explain why this intuition regarding the Allee effect and the scarcity of mate-finding sites may be in error. We begin from the position that the encounter probability of a male and female at a mating location can be viewed as a variant of the birthday paradox: the calculation of the probability that at least two people in a group of randomly selected individuals will share the same birthday (Feller 1957). This probability is of importance when calculating quantities in widely various applications such as the probability that airplanes will collide, or that there are matches between random length clone fragments in DNA mapping (Soderlund et al. 2000), and has been used in the past to estimate fish population sizes (Schnabel 1938). The birthday paradox is not a true paradox; however, the finding that a group of only 23 people is required to have a greater than $50 \%$ probability that two share a birthday is a surprise to many. This difficulty with intuition most likely relates to the fact that the birthday paradox asks whether any of the people in a given group has a birthday matching any of the others, not one in particular. As a result, in this small group of people, there are over two hundred combinations to consider.

In the following, we examine how the use of a small number of landmarked sites might impact the distribution of individuals, and therefore the probability of mate-encounter. We begin by comparing the expected distribution of individuals which have an aggregative tendency with the expected distribution for individuals using only landmarking. We use a calculation similar to that of the birthday problem to determine the probability that at least one mate encounter could occur for a given number of landmarked sites. However, since we have two sexes, it is necessary to use a modification of the birthday problem where there are two classes of individuals (Wendl 2003; Nakata 2008). Using this modification, we consider the effect of sex ratios on the mate encounter probability, and the impact of different mating strategies (monogamy vs. polygyny) on the estimated number of mated females. Since the combinatorially exact calculations require lengthy computations, we also evaluate a number of different approximation methods for estimating the number of mated females.

Unlike the birthday problem where all individuals must have a birthday, not all individuals may arrive at a landmarked site. Although many species travel long distances to arrive at landmarked sites (Skevington 2008), some sites may be too distant for detection, or individuals may inaccurately identify suitable sites. For example, Pe'er et al. (2004) found that butterflies in the vicinity of multiple peaks typically select the highest within visual range (approximately $50 \mathrm{~m}$ ). We should expect that mating probability will be reduced if only a small proportion of the sexually active population manages to arrive at landmarked sites. Therefore, we also examine the impact of site-finding success rates on the predicted number of mated pairs.

Finally, using these approximations and simulation data, we examine the potential impact of the landmarking strategy on the strong Allee threshold. For some applications, such as predicting the risk of invasion, an impact of landmarking on a strong Allee threshold could be relevant for determining whether a given management strategy is feasible.

\section{Modelling the effect of landmarked mate-finding sites on Allee thresholds}

Attraction to conspecifics versus random assortment among landmarked sites

We begin our exploration of the impact of a landmarking strategy on mate encounter by first noting that there is a difference in the expected distribution of individuals where there is a tendency to be attracted to higher densities of conspecifics (e.g., the mating aggregations mentioned by 
Gascoigne et al. (2009)), versus only attraction to landmarked features. If individuals are equally attracted to all landmarked sites and have a reasonable probability of travelling to any site, they will be less aggregated, and have a lower probability of finding a mate than when there is strong attraction to high-density groups of conspecifics. However, for either strategy, we expect a larger number of male-female pairs when there are fewer mate encounter sites.

When individuals settle at random over a finite number of landmarked sites, their final distribution is best described as uniform. For example, to simulate one instance of an expected distribution of 8 males and 8 females across either 5 or 15 mate-finding sites, we draw 8 values twice from a uniform distribution with the given number of possible values (Fig. 1a, d) (we completed this, and all other calculations, in MATLAB version R2013a). For 15 landmarked locations, most sites will have one or two individuals. However, for a smaller number of sites, there is a higher probability that males and females end up at the same location (compare Fig. 1a, d).

An aggregative tendency will produce higher local densities of individuals. If males and females respond directly to the density of conspecifics (but not the sex-ratio), we can simulate their distribution across the mate-finding sites using two sets of 8 random numbers drawn from the negative binomial distribution (Boswell and Patil 1970). Depending on the strength of the attraction, individuals

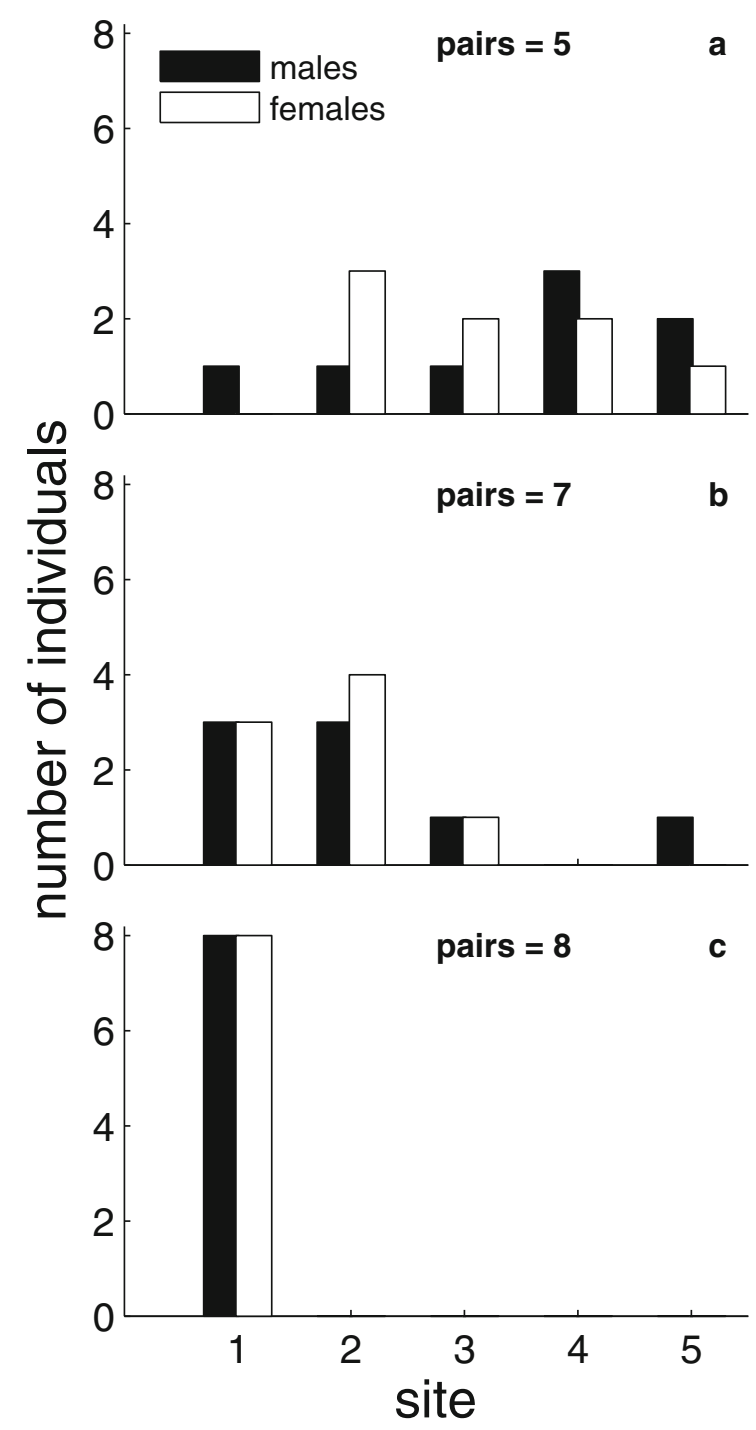

Fig. 1 Examples of the distribution of 8 males and females, and the total number of male-female pairs ('pairs') summed across all locations for $5(\mathbf{a}-\mathbf{c})$ or 15 sites $(\mathbf{d}-\mathbf{f})$ as given by the uniform distribution
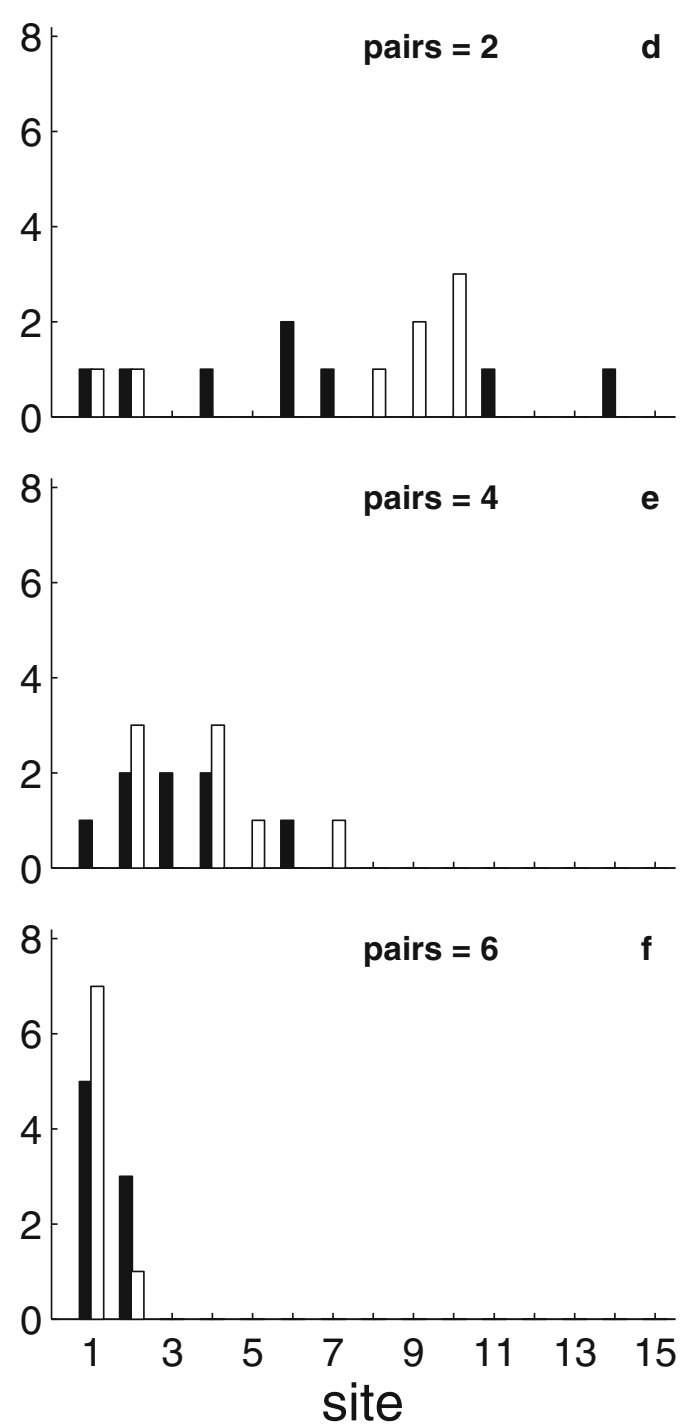

(a, d), the negative binomial distribution with low aggregation (b, e), or the negative binomial distribution with high aggregation $(\mathbf{c}, \mathbf{f})$ 
will collect at one or very few sites, and consequently nearly every individual may find a mate (Fig. 1b, c). However, when there are more possible sites, the probability of males and females occupying the same location is somewhat reduced even with this aggregative tendency (compare Fig. $1 b$ with e, and c with f).

In summary, when individuals are not attracted to higher densities of conspecifics, their distribution is predictably less aggregated (compare Fig. 1a with $c$ and b). Therefore, the assumption that individuals are only attracted to landmarked sites and not conspecifics will lead to lower estimates for the probability that individuals will successfully find a mate. It is this more conservative scenario that we address in the following work, but we note that the positive impact of fewer mating sites on mating probability will be similar for species with conspecific attraction, although it will be of much smaller magnitude.

\section{The probability of at least one successful mating}

If mate-finding sites such as hilltops, tall trees or large rivers have long range detectability, and are within reasonable travel distance, then the main barrier to a successful reproductive event is that a male and female will be present at the same location. Here, we calculate the probability of at least one mate encounter for a given number of landmarked sites, and numbers of males and females. This probability has some useful interpretations. When developing policies regarding a potentially invasive species, if even a single mating is quite unlikely for a given density, then the potential invasive may represent very low risk. Similarly, an endangered species is probably beyond all recovery if even a single mating becomes quite unlikely.

The encounter probability of a male and female individual at a mating location can be viewed as a variant of the birthday paradox. However, rather than just calculating the probability that two individuals will share a category as in the birthday paradox, we have two kinds of individuals in our problem: males and females. To address this complication, we use more recent work that addresses the probability that two different kinds of objects end up in the same bin (Wendl 2003; Nakata 2008). To apply this probability to mate-finding, we consider that our two kinds of objects are males and females, and the bins are those locations where individuals go to seek mates (e.g., spawning rivers, hilltop sites). If we assume that encounters between males and females are unproblematic once at a mate-finding site, and furthermore, that individuals are ready for mating at about the same time, we can show that the probability of at least one mate encounter is quite likely at very low population density.

It is easiest to calculate the probability that there are no males and females in the same mating location $\left(P_{0}\right)$, and then use one minus this probability $\left(1-P_{0}\right)$ to determine the chance that there is at least one mate encounter. Following Wendl (2003), we can calculate the exact probability that there are no simultaneous occurrences of male and female individuals in the same mate-finding location as:

$P_{0}(m, f, r)=\frac{1}{r^{m+f}} \sum_{i=1}^{m} \sum_{j=1}^{f} S_{2}(m, i) S_{2}(f, j) \prod_{k=0}^{i+j-1} r-k$

where $m$ is the number of males, $f$ is number of females, $r$ is the number of mate-finding sites, and $S_{2}$ refers to Stirling numbers of the second kind. So, $S_{2}(n, k)$ represents the number of ways we can partition $n$ individuals into $k$ groups. We assume that the distribution of males is independent of the distribution of females, so that $r^{m+f}$ gives the total number of ways of arranging the males and females among $r$ landmarked sites. When considering the probability of no encounter events, we are interested in the number of these arrangements that result in no female arriving at a site with a male. For example, there are 16 ways to arrange 2 males and 2 females across 2 sites, but only 2 of these arrangements isolate females from males (see Appendix A). The probability that there is at least one meeting of a male and female in the same location is then given by $1-P_{0}$.

Where there is a $1: 1$ sex ratio, the probability of at least one matched male and female pair in a landmarked location (monogamous mating) increases as the population size increases. However, the highest probabilities for any given population size are found where there are the fewest landmarked sites (Fig. 2a). These probabilities are not unique. That is, a different number of males and females can also yield similar probabilities. Where the number of individuals is held constant, the highest probabilities are obtained at a $1: 1$ sex ratio, and are reduced by a reduction in the number of males or females. However, it is only when the sex ratio is significantly skewed that we see substantial reductions in these probabilities (Fig. 2b).

\section{Estimated number of matings}

We have estimated the probability of at least one mating in a small population using a landmarking strategy. For potentially invasive species, if the probability of mate encounter is very small over the probable range of introduction densities, our management concerns may end there. If, however, there is a reasonable chance of a single mating (say greater than $50 \%$ ), the next question to ask is how many mated females should we expect.

An exact calculation for the expected number of matched monogamous pairs (or the related measure, the probability that a female will be mated) was offered by Wendl (2005), in the context of DNA fingerprinting. In our context, the number of mated monogamous pairs is $k=\sum_{i=1}^{r} \min \left(m_{i}, f_{i}\right)$, 


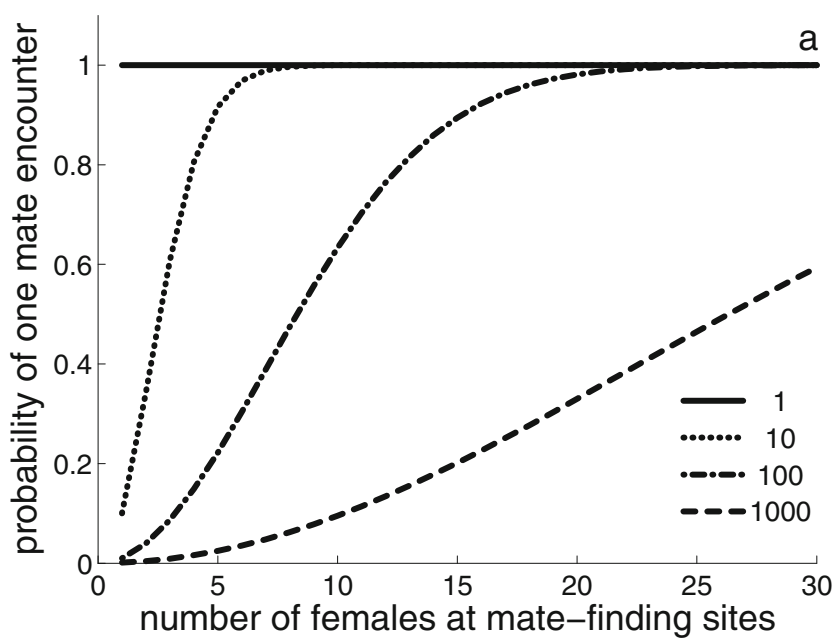

(1:1 sex ratio)

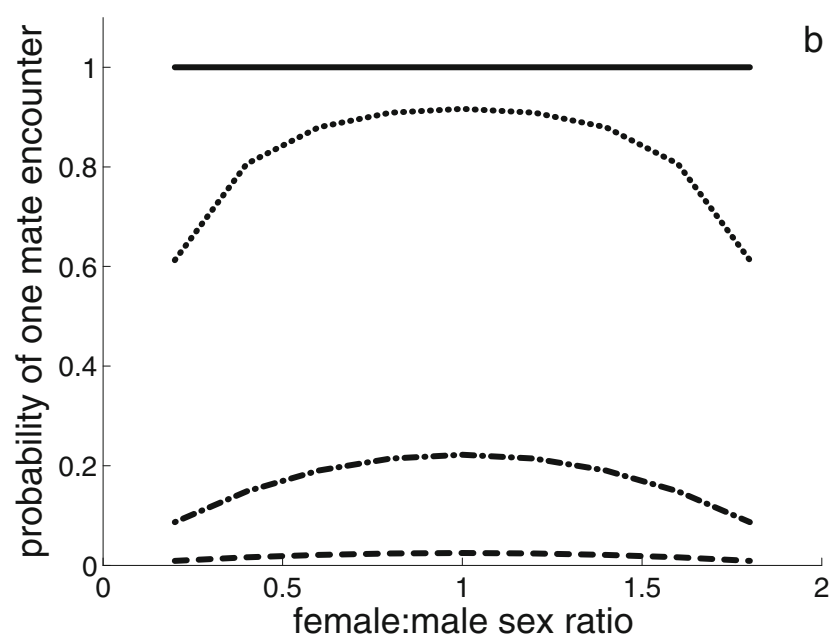

Fig. 2 The probability of a male and female occupying the same matefinding site vs. the number of females where there is 1:1 sex ratio (a), and the probability of a male and female occupying the same site vs. the ratio of females:males where there are 10 individuals (b), for 1, 10, 100 or 1000 landmarked sites (solid, dotted, dashed-dotted and dashed lines, respectively)

where $m_{i}$ and $f_{i}$ are the number of males or females at location $i$, and there are $r$ total landmarked locations. As noted by Wendl (2005), the exact solution to the problem rests on a calculation of the number of ways the sets of all males and all females can be partitioned into groups, and these groups of different sexes distributed across the landmarked sites. This exact calculation is quite lengthy, and for an intermediate to large number of landmarked locations, takes considerably more computer processing time than a simple simulation. Moreover, there is excellent agreement between simulation and the exact calculation (see Appendix B). Therefore, we report the simulated results here.

Over 100,000 replicate simulation trials, the expected number of matched pairs for a given population density decreases as the number of mate-finding sites increases (Fig. 3a). For example, for 50 males and 50 females distributed across 2 landmarked locations, summing over $i$, we expect 46 mated pairs, whereas for 20 possible locations, we expect only 33. Instead of monogamous pairing, we could assume that one male can fertilize all females at his location (complete polygyny). In this case, the maximum expected number of mated females is a higher proportion of the number of females at mate-finding sites than in monogamous mating, but the same general relationships apply.

\section{Probability of site-finding}

The previous calculation is based on the total number of individuals in a population and assumes that all individuals arrive at a landmarked site. Here, we investigate
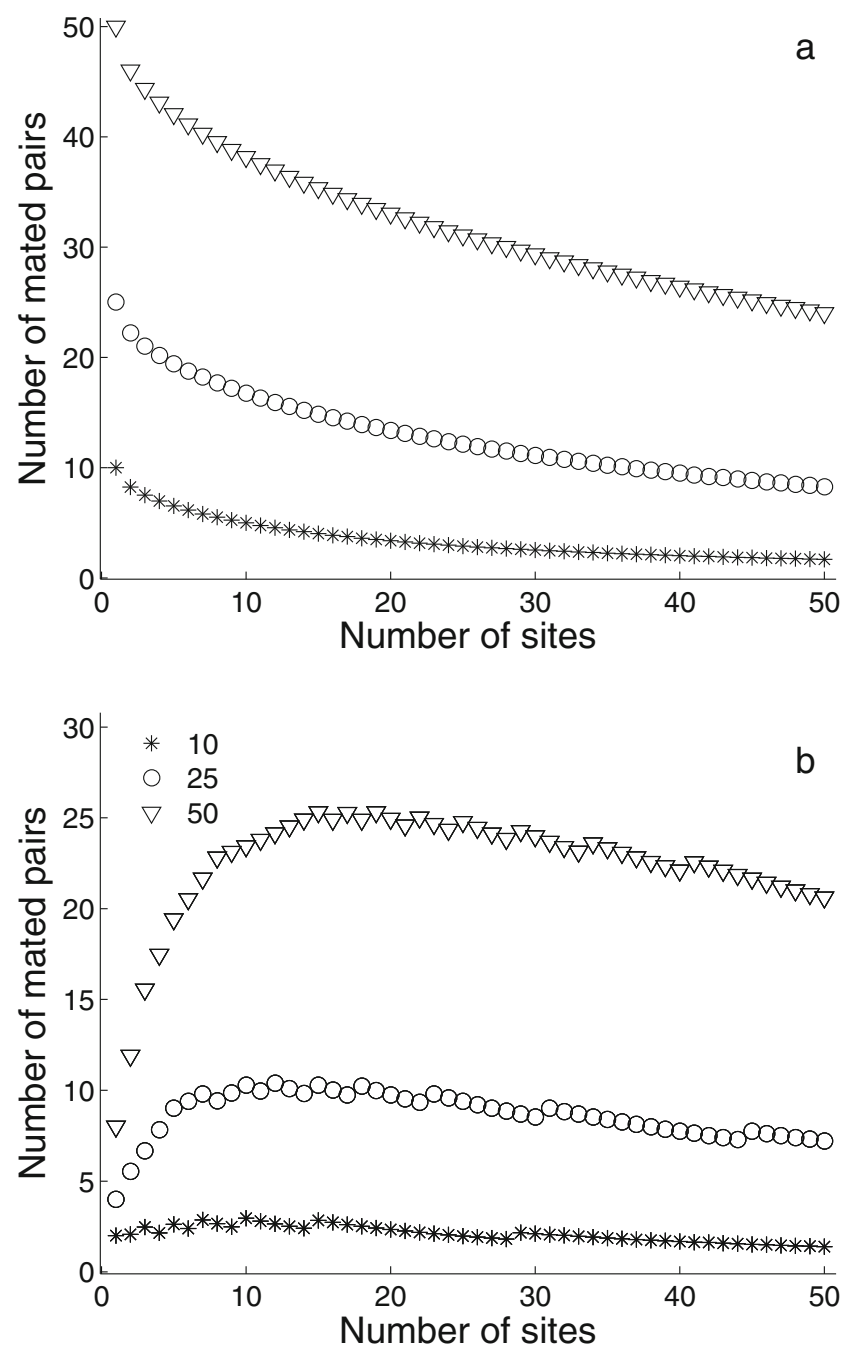

Fig. 3 The expected number of mate pairs when all individuals in the population (or some fixed percentage of these individuals) locate landmarked sites (a) or when the probability of an individual locating a site increases with the number of sites, $r$ (as $\frac{r}{r_{0}+r}$, where $\left.r_{0}=5\right)(\mathbf{b})$, when there are 10, 25 or 50 females and males (star, open circle, open triangle, respectively) 
the consequences of relaxing that assumption for the predicted number of monogamous mated pairs. It is certainly possible that only a portion of the population is successful at locating landmarked sites. For example, not all fish present in the lake may arrive at suitable spawning rivers, or fish may incorrectly identify rivers as suitable. If some individuals do not actively seek out mate-finding sites or if the landmark cues are imperfect and lead to the selection of an unsuitable location, then the number of individuals that are matched with a site will be smaller, and the probability of finding a mate, given as a function of total population density, will be lower.

One simple way to approach difficulties in locating landmarked sites is to multiply our total number of sexually active adults by a given probability of encountering an appropriate location during the correct environmental conditions. If we take a low but not restrictive value of $20 \%$ of the population meeting this criteria, then we can use the previous calculation to determine the probability of one successful encounter. If $20 \%$ of a population of 50 males and 50 females (or $1 \%$ of a population of 1000 males and 1000 females, $10 \%$ of a population of 100 males and 100 females, etc.) were successful at finding mating locations, then the predicted number of successful pairs would be the same as the probability based on the expected number of 10 individuals of each sex that arrive at landmarked sites (Fig. 3a).

If, however, the total number of individuals that arrive at any landmarked site is a positive function of the number of locations, the situation may change. For example, we could use a saturating function to describe the probability that an individual arrives at a mate-finding site. The function $f(r)=r /\left(r_{0}+r\right)$ ranges from 0 to 1 , where the value of the function increases to the asymptote of 1 as $r$, the number of landmarked sites, increases. The number of individuals that successfully locate a site is then calculated as the number of individuals in the population multiplied by the value of $f(r)$. In this case, the probability of one successful encounter at low population size can be highest for an intermediate number of mate-finding sites. As a result, we expect a larger number of mated pairs at an intermediate number of sites (the position of the peak will be determined by the half-saturation constant). That is, the difficulties of arriving at a mate-finding site when there are few of these sites will be offset by the problem of few or no individuals at each location when the number of sites is very large (Fig. 3b). This result depends on the value of the half-saturation constant, $r_{0}$. As $r_{0}$ approaches zero, we regain our original result (Fig. 3a), but as $r_{0}$ becomes large, a larger number of mate-finding sites are required to have some significant probability of one successful encounter at low density.
Approximating the effect of landmarking strategies on mate-finding

These exact solutions, or equivalent simulated solutions, are cumbersome for calculations regarding population dynamics. We would like simpler formulas to approximate the exact solutions for the expected number of mated females. For monogamy and a one to one sex ratio, one of the most commonly used equations to predict the probability that a female will find a mate is $m /(m+\theta)(1)$, where $m$ is the number of males in the population and $\theta$ expresses factors that determine the search success (e.g., Dennis 1989; Veit-and-Lewis 1996). In other work (Cuddington et al. 2014), we have also used a normal approximation as: $m-$ $\sqrt{m(\theta-1) / \pi}(2)$, where again we assume an equal number of males and females (see derivation and more general cases in Walter (1980)). For polygyny, $1-e^{-m / \theta}$ (3) is often used (e.g., Dennis 1989; Hopper and Rousch 1993), but we could also use a binomial approximation, where the probability of a female mating is $1-(1-1 / \theta)^{m}(4$; see derivation in Appendix A). For our landmarking strategy, in all these equations, $\theta$ is the number of mate-finding locations, $r$.

Unlike the exact solution (Appendix B), to approximate the number of mated pairs, we assume that the probability of one female mating is independent of the probability that another female mates. So, to calculate the expected number of mated females, $E(P)$, we simply multiply this probability by the number of females. However, if not all males and females locate a site, we need to correct values of both $m$ and $f$ so that they express the number of males or females that locate mate-finding sites rather than total number of each sex in the population. For example, where site-finding is a saturating function of the number of sites, $r$, the actual number of males, $m$, at landmarked sites will be $m_{\text {sites }}=m_{\text {total }} * r /\left(r+r_{0}\right)$.

In general, it is easiest to obtain a reasonable approximation for polygynous mating because the conditional nature of the problem is reduced (i.e. the presence of another female at a site with a male does not lower the probability that a given female will also mate, as it does in the case of monogamy). Of the four approximations investigated, when all individuals locate a site and are monogamous the saturating function (1) and Walter's normal approximation (2) have a similar range of error. However, both approximations are biased. In a set of 100,000 replicate simulations, the equation $m /(m+\theta)$ performs reasonably well but will generally overestimate the mean number of pairs (maximum overestimate 3.57 , minimum underestimate 0.98 , Fig. 4a),except in the case of a single mate-finding site. Walter's normal approximation will consistently underestimate the number of mated females (maximum overestimate 0 , minimum underestimate 4.33, Fig. 4b). However, neither 


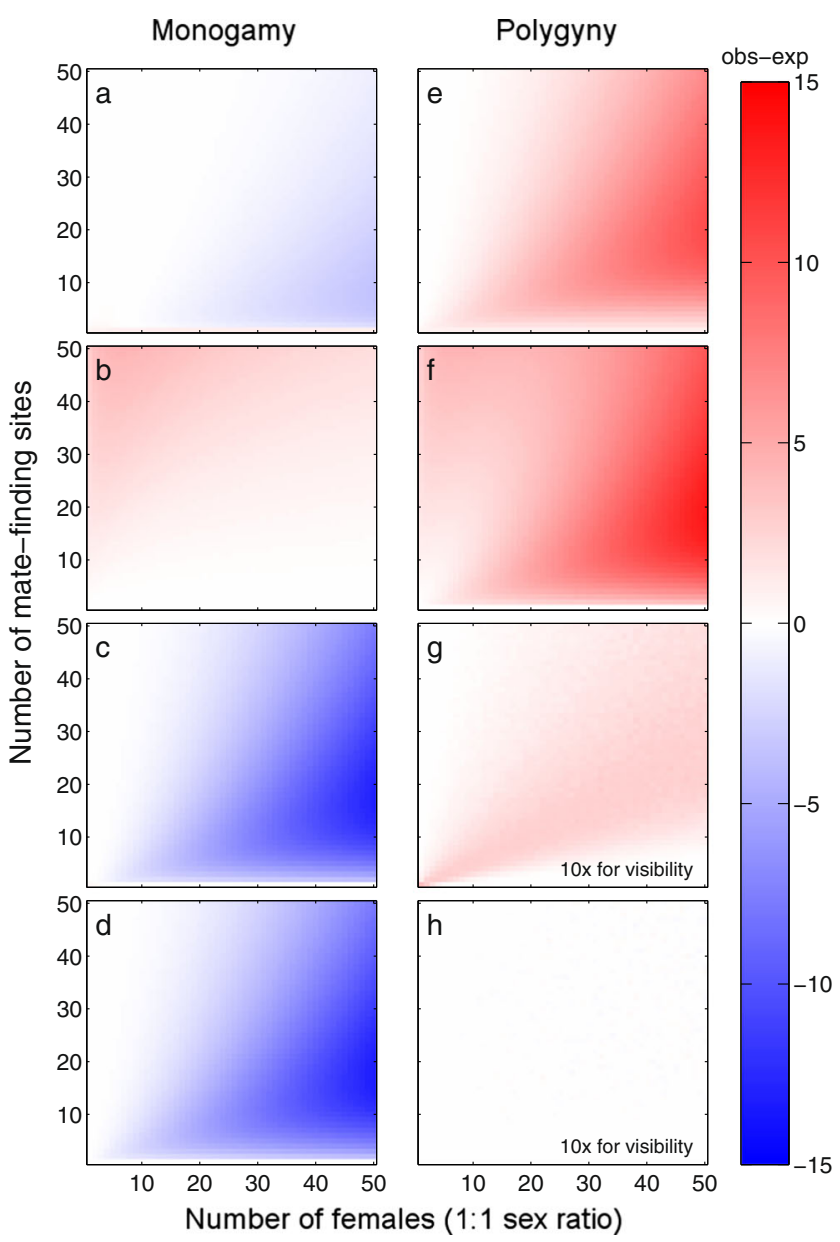

Fig. 4 The difference between the mean number of mated females observed via simulation and the number predicted by: Eq. 1 (a, e), Eq. 2 (b, f), Eq. 3 (c, g) and Eq. 4 (d, h), for either monogamous mating (a-d) or complete polygyny (e-h). Differences were multiplied by 10 in panels $\mathbf{g}$ and $\mathbf{h}$ to aid visualization

of these approximations is appropriate for polygyny (Fig. $4 \mathrm{e}, \mathrm{f})$.

Unsurprisingly, given the strongly conditional nature of the exact solution, the poisson (3) and the binomial approximations (4) are not good for monogamy, producing a consistent overestimate ranging up to 13 pairs (Fig. 4c, d). However, for polygyny, these two approximations perform the best of the four investigated and are only slightly biased to underestimate the number of mated females. The binomial approximation has a modestly superior performance (maximum overestimate 0.034 , minimum underestimate 0.069 , Fig. 4h), compared to the poisson approximation (maximum overestimate 0.001 , minimum underestimate 0.37 , Fig. $4 \mathrm{~g}$ ).

\section{Threshold for strong Allee effect}

Finally, we can describe how the landmarking strategy might affect population dynamics by determining its impact on a strong Allee threshold. If the per capita population growth rate is given by the number of births minus the number of deaths as $(b E(P)-d N) / N$, where $E(P)$ is the expected number of mated females estimated as above, $b$ is the recruitment rate per mated female, and $d$ is the per capita death rate, then we can solve for the population density at which this rate is equal to zero to generate a function describing how the Allee threshold changes with the number of landmarked sites (in practice, these functions are generally implicit equations).

Populations with the lowest number of landmarked sites have the lowest strong Allee threshold when all individuals in a population, or a fixed proportion of the population, arrive at a site (Fig. 5a). If the total number of individuals that arrive at mate-finding sites is a saturating function of the number of locations, then the lowest Allee threshold will be found for an intermediate number of landmarked sites. Where the half-saturation constant, $r_{0}$, is small, the Allee threshold is extremely large for very few sites, but drops rapidly for an intermediate number of sites (Fig. 5b). Of course, when the proportion of the population that locates
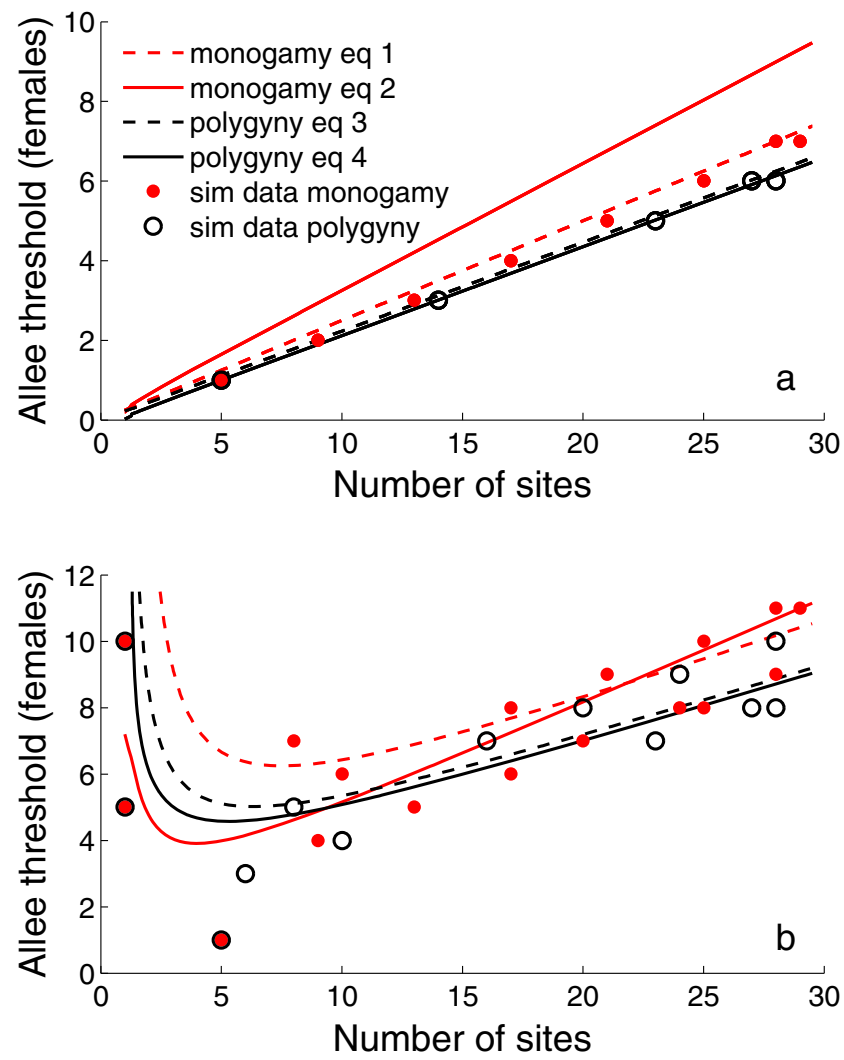

Fig. 5 Strong Allee threshold estimated by one of the four equations given in the text, or as given by 100,000 simulations, for the cases where all individuals locate a site (a) and where the number of individuals in the population that locate mate-finding sites is a saturating function of the number of sites $\left(r_{0}=5\right)(\mathbf{b})$ 
landmarked sites increases quite slowly as the number of sites increases (large $r_{0}$ ), the number of sites that produces the minimum Allee threshold will increase.

\section{Discussion}

Many species may employ strategies that mitigate matefinding component Allee effects via a restriction on the number of locations where individuals search for mates (e.g., Shields 1967; Wickman 1988; Mills 1989). The use of particular 'landmarked' mate-finding locations sometimes can be distinguished from the occurrence of mating in areas that are likely to boast nesting areas and resources for successfully rearing offspring. The best known instance of this behaviour is hilltopping in butterfly species where males and females use topographical cues to aggregate at areas of high elevation (e.g., Pe'er et al. 2004). Hilltops are not the only features used for this strategy. For example, using plastic spruce tree models of different heights, Wickman (1994) demonstrated that Coenonympha pamphilus male butterflies and virgin females were attracted to the tallest trees in an open grassland. Skevington (2008) defines a landmark mating strategy as setting up mating aggregations over any landscape feature, where such features can include a tuft of grass, a stream course or a hilltop. These areas do not necessarily have host plant species for either larval or adult life stages. It is generally accepted that the use of these areas is a behavioural mechanism to increase mate encounter rate (Shields 1967; Scott 1970), and recently sophisticated simulation models have corroborated the theory that hilltopping can improve butterfly mating success at low density (Pe'er et al. 2006; Painter 2014). In other systems, the use of a restricted number of mating locations may be combined with access to resources necessary for successful maturation of offspring (e.g., brook trout, see Purchase and Hutchings 2008). In addition, some mating behaviours, such as lekking, are primarily examined in light of questions regarding sexual selection (e.g., bower-building cichlid fish see Young et al. 2009) but may have impacts on mate encounter probability.

All these landscape-related strategies may have the effect of increasing the probability of mate encounter over what would be expected during the non-breeding distribution of individuals. Our calculations suggest that such behaviours are an extremely efficient way to increase mate encounter when there are relatively few such sites. Attraction to landmarked sites will never produce the same concentration of density as strong attraction to high density of conspecifics. Nonetheless, when there are few such sites, landmarking may be a highly efficient method of clustering individuals. Indeed, landmarking is potentially more efficient than conspecific attraction that produces mating aggregations, which is disrupted at very low density (e.g., Rowe and Hutchings 2003). A combination of landmarking and attraction to conspecifics will be even more efficient.

We find that a landmarking strategy can lead to high mate encounter probability at low density, which has some unexpected management implications for both invasive and endangered species. In general, the fewer the number of landmarked locations, the larger the probability of at least one mating. Therefore, the expected number of mated females is greatest, and the strong Allee threshold is smallest, for the fewest number of landmarked sites. Superficially, this finding may seem counter-intuitive, but it is well known that intuitions about probability and risk are not always reliable (e.g., Kahneman et al. 1982). This seems to be particularly true when our probability calculation involves combinatorics: a counting up of the number of ways in which an event can occur. For example, managers may believe that if a small number of Asian carps escape into a Great Lake, such as Erie, they are unlikely to establish a population because it will be difficult to find a mate. Our calculation, using a variant of the birthday paradox, demonstrates that this intuition is probably false. Indeed, using a spawning-river structured model, Cuddington et al. (2014) found that the limited number of suitable rivers increased the probability of a successful Asian carp invasion.

Of course, not all individuals may be successful at finding a landmarked mating site. We expect that species invading new areas may have difficulty locating or identifying suitable sites. Butterflies may select the highest hilltops within visual range (Pe'er et al. 2004), and therefore may not locate higher, but more distant peaks. Asian carp can be triggered to spawn by warm water temperature and high flow rate (Costa-Pierce 1992; Kolar et al. 2005), but may be unable to determine if a river is of sufficient length for successful reproduction. It could be that only some small percentage of individuals find a suitable landmarked site, in which case the probability of a single mating event is somewhat reduced, but is still highest for the smallest number of landmarked sites. Alternately, the probability of finding a suitable location could increase at a decreasing rate with the number of such sites. In the latter case, the probability of at least one successful mating when the population size is small will also depend on how quickly search success increases with the number of locations. In general, the largest probability will be found for an intermediate number of landmarked sites (Fig. 4) and as a result, population growth will be positive for an intermediate number of sites, rather than for a larger number, as intuition might suggest (Fig. 5).

However, as we note, some landmarked sites may be more accessible or more detectable than others, and we did not explicitly include this factor in our analysis. Differing accessibility is different than some sites being preferred 
to others when all have equal accessibility. In the case of simple preference, a larger number of individuals will collect at the highly preferred sites, and mate-finding would be more probable (i.e., similar to the case when individuals are attracted to high densities of conspecifics, Fig. 1). In the case of differing accessibility or detectability, it may be more difficult to travel to or to locate some particular sites. For example, when a non-native species is introduced, very few individuals may reach sites distant from the initial point of introduction. In this case, the accessibility of sites would change through time as the individuals reproduced and spread through the landscape. Therefore, we suggest that initially, there would be a greater probability of at least one mating, as individuals would make use of the smaller number of mate-finding sites in their immediate vicinity.

The ratio of females to males in the population can also alter the probability of at least one successful mating (Fig. 2), but does not have a large effect until sex ratios are seriously skewed. Of course, males and females may arrive at a mate-finding location at different times. If individuals will only wait to encounter the opposite sex in a given location for a small time interval, the probability of successful mating will be further reduced. However, even for this scenario, a simple calculation using Wendl's formula (2003) shows that the probability of at least one successful mating is still quite large (e.g., $75 \%$ for 10 females and 10 males and 60 time/space intervals). Of course, if individuals only briefly visited mate-finding sites, and there were an even larger number of mating sites, this probability would be reduced (i.e. we would have a very large number of space/time intervals). However, in some species, males linger in the landmarked location for longer periods of time, which would of course increase mating success (e.g., Rutowski 1991).

Simulations provide an extremely good approximation to the expected number of mated females in the scenarios we investigated, and are quite useful given the lengthy calculations required for the exact solutions (Appendix B). However, simpler equations can also be used to approximate the exact solutions, and some are reasonably accurate for polygyny. The standard equations we tested for monogamy over- or under-estimated the number of mated pairs in particular ranges. When using such approximations, we need to be cognisant of the direction of bias. For example, a commonly used estimate from the literature $(1: m / m+\theta)$ when applied to landmarking will consistently overestimate the number of mated pairs when there is modestly high population size and few sites. However, this approximation is more accurate for low population size and a larger number of sites. As a result, we suggest the use of this equation when generating optimistic estimates of extinction risk. Walter's (1980) normal approximation
(2) underestimate the number of pairs at low population density and many sites. Therefore, the normal approximation would be an optimistic estimate of risk for potentially invasive populations, and a pessimistic estimate of extinction risk.

When attempting to determine if a demographic matefinding Allee effect occurs for endangered or invasive populations, we need to be careful when the organisms in question use a restricted number of mate-finding locations. This is particularly true because mechanisms such as true aggregation are disrupted at low density, while attraction to landmarked sites is unaffected by density. Conservation programs may take advantage of the mate-finding at landmarked sites. Reintroduction efforts typically rely on the release of a sufficient number of individuals such that enough will survive to breed and re-establish a population (Rakes et al. 1999; Daugherty et al. 2008). Our analysis suggests that for some species (e.g., salmonids, sturgeon), this effort may be hampered by the creation of too much, overly fragmented, mating habitat. A large number of small and diffuse mating locations could require the release of a larger number of individuals to achieve positive population growth. Fewer mate-finding locations could initially concentrate individuals and thus maximize mate-pairings and fertility at lower release densities. Of course, the use of such a strategy must be balanced against the potentially large negative consequences that might ensue when environmental stochasticity or human impacts impinge on one of a few landmarked sites. Given the risks, clearly one would want experimental data that confirms much higher mating success with fewer landmarked sites before implementation.

Estimation of risk of establishment of non-native species, and management of established invasive species, is often predicated on the presence of Allee effects. For example, the release of sterile males in biological control (e.g., Knipling 1955; Twohey et al. 2003) is aimed at reducing the number of successful matings, and therefore, pushing the per capita population growth rate to zero. While this type of strategy is undeniably useful for many species, we urge caution when applying this reasoning to species that use a restricted number of mate-finding sites. The use of landmarked sites does not necessary produce large densities of individuals at a given location, but it can certainly increase the probability that a male and female meet, even when there is no prior knowledge of particular landmarks. Certainly, landmarking species can be successful invaders (e.g., Braby et al. 2014). Our examination of this issue suggests that when a strong Allee threshold exists for these types of species, it may be very small indeed, and unlikely to be reached via management aimed at reducing the number of viable males. Moreover, control actions that focus on reducing suitable mating habitat in an effort to reduce reproduction may have the opposite of the intended effect depending on search 
strategies and the current number of landmarked locations. We conclude that such 'landmarking' is a frequently overlooked mating strategy that should be accounted for when predicting invasion risk.

Acknowledgments This work was supported by Fisheries and Oceans Canada.

Open Access This article is distributed under the terms of the Creative Commons Attribution License which permits any use, distribution, and reproduction in any medium, provided the original author(s) and the source are credited.

\section{Appendix A}

To calculate the exact probability that there are no mated females let $E_{i}$ be the event that a female $i$ goes to a matefinding site that contains no males, and therefore $P\left\langle E_{1} \cap\right.$ $\left.E_{2} \cap . . E_{f}\right\rangle$ is the probability that no female has a male at her site where there are $f$ females. It is difficult to compute $P\left\langle E_{i}\right\rangle$ without knowing how many sites are empty of males. The probability that a site contains no males depends on the number of possible ways $m$ males can be arranged across $r$ sites, and the number of sites that are empty for each of these arrangements. So for example, for two males and two sites, there are four possible arrangements of males (male $\mathbf{a}$ at site 1 , and male $\mathbf{b}$ at site 2 , male $\mathbf{b}$ at site 1 and male $\mathbf{a}$ at site 2 , both males at site 1 , or both males at site 2 ). Out of these arrangements, two lead to an empty site. The female can be found at only one of the two sites for each arrangement of males, so that there are eight possible arrangements of males and females. Only two of these arrangements results in isolation of the female, so there is a $25 \%$ probability of no mating. If there are two females, then the probability that neither female ends up at a site with males is $12.5 \%$ (Fig. 6).

In the main text, we use the equation derived by Wendl (2003) for DNA fragment matches to provide an exact calculation of the number of arrangements of males and females over a given number of landmarked sites that result in no matings. If we are only interested in the probability of any matings, there is little computational advantage in using an estimate rather than this exact calculation. However, obtaining the total estimated number of matings is quite onerous because of the long calculation required to determine the number of possible arrangements of individuals across the landmarked sites that result in a given number of mated pairs (see Appendix B). For example, with only two males, two females and two sites, there are eight arrangements that lead to one mating and six arrangements that yield two matings (Fig. 6). If we are interested in the number of mating events, then an approximation of mating probability would be quite beneficial.

\begin{tabular}{|c|c|c|c|c|}
\hline & $\mathrm{M}_{1} \mathrm{M}_{2}$ & $\mathrm{~F}_{1} \mathrm{~F}_{2}$ & $\mathrm{~F}_{1} \mathrm{~F}_{2}$ & $\mathrm{M}_{1} \mathrm{M}_{2}$ \\
\hline \multicolumn{5}{|l|}{$k=1, H=8$} \\
\hline & $\begin{array}{l}\mathrm{M}_{1} \mathrm{M}_{2} \\
\mathrm{~F}_{1}\end{array}$ & $\mathrm{~F}_{2}$ & $\begin{array}{l}\mathrm{M}_{1} \mathrm{M}_{2} \\
\mathrm{~F}_{2}\end{array}$ & $\mathrm{~F}_{1}$ \\
\hline & $F_{1}$ & $\begin{array}{l}\mathrm{M}_{1} \mathrm{M}_{2} \\
\mathrm{~F}_{2}\end{array}$ & $\mathrm{~F}_{2}$ & $\begin{array}{l}\mathrm{M}_{1} \mathrm{M}_{2} \\
\mathrm{~F}_{1} \\
\end{array}$ \\
\hline & $\begin{array}{l}\mathrm{M}_{1} \\
\mathrm{~F}_{1} \mathrm{~F}_{2}\end{array}$ & $\mathrm{M}_{2}$ & $\begin{array}{l}\mathrm{M}_{2} \\
\mathrm{~F}_{1} \mathrm{~F}_{2}\end{array}$ & $\mathrm{M}_{1}$ \\
\hline & $\mathrm{M}_{1}$ & $\begin{array}{l}\mathrm{M}_{2} \\
\mathrm{~F}_{1} \mathrm{~F}_{2} \\
\end{array}$ & $\mathrm{M}_{2}$ & $\begin{array}{l}\mathrm{M}_{1} \\
\mathrm{~F}_{1} \mathrm{~F}_{2} \\
\end{array}$ \\
\hline \multicolumn{5}{|l|}{$k=2, H=6$} \\
\hline & $\begin{array}{l}\mathrm{M}_{1} \\
\mathrm{~F}_{1}\end{array}$ & $\begin{array}{l}\mathrm{M}_{2} \\
\mathrm{~F}_{2}\end{array}$ & $\begin{array}{l}\mathrm{M}_{2} \\
\mathrm{~F}_{1} \\
\end{array}$ & $\begin{array}{l}\mathrm{M}_{1} \\
\mathrm{~F}_{2} \\
\end{array}$ \\
\hline & $\begin{array}{l}\mathrm{M}_{1} \\
\mathrm{~F}_{2} \\
\end{array}$ & $\begin{array}{l}\mathrm{M}_{2} \\
\mathrm{~F}_{1} \\
\end{array}$ & $\begin{array}{l}\mathrm{M}_{2} \\
\mathrm{~F}_{2} \\
\end{array}$ & $\begin{array}{l}\mathrm{M}_{1} \\
\mathrm{~F}_{1}\end{array}$ \\
\hline & $\begin{array}{l}\mathrm{M}_{1} \mathrm{M}_{2} \\
\mathrm{~F}_{1} \mathrm{~F}_{2} \\
\end{array}$ & & & $\begin{array}{l}\mathrm{M}_{1} \mathrm{M}_{2} \\
\mathrm{~F}_{1} \mathrm{~F}_{2} \\
\end{array}$ \\
\hline
\end{tabular}

Fig. 6 The number of possible arrangements $(H)$, resulting in a given number of matings $(k)$ of two males, and two females across two mating sites

The most straightforward approach is to use a binomial approximation and assume that events are independent. Consider a specific female $j$ and a specific male $i$ each of which are arbitrarily selected from a given number females, $f$, and a number of males, $m$, respectively. Further assume that the location of female $j$ has been determined. Then, we want the probability of the event $E_{i j}$ that the location of the male $i$ will match the location of the female $j$. Since $i$ can occur at any one of $r$ sites with equal probability, we have $P\left\langle E_{i j}\right\rangle=\frac{1}{r}$ and the probability that the male $i$ is not at the same location as the female $j$ is simply the complement $P\left\langle E_{i j}^{c}\right\rangle=1-\frac{1}{r}$.

Now, we can expand this calculation to compute the probability $p_{0}$ that no other males are found at the same location as the female $j$. This probability would be the intersection of all individual trials where the event $E_{i j}^{c}$ occurs, so $P\left\langle E_{1 j}^{c} \cap E_{2 j}^{c} \cap E_{3 j}^{c} \ldots \cap E_{m j}^{c}\right\rangle$. Since the males are independently distributed among the mating sites, the probability that this female does not find a mate as given by this approximation is simply $p_{0}=P\left\langle E_{1 j}^{c}\right\rangle * P\left\langle E_{2 j}^{c}\right\rangle * P\left\langle E_{3 j}^{c}\right\rangle \ldots *$ $P\left\langle E_{m j}^{c}\right\rangle=\left(1-\frac{1}{r}\right)^{m}$. Readers will note that this is the binomial probability density function $\left(\begin{array}{l}m \\ x\end{array}\right) p^{x}(1-p)^{m-x}$ for the case where $x=0,(1-p)^{m}$.

The performance of this binomial approximation varies with the number of landmarked sites and the number of 
individuals, which determine the dependence of event probability. This dependence will be larger for a small number of mating sites. Consider again the probability of no matings between 2 males and 2 females distributed across two sites. There are 16 possible arrangements of the individuals and 2 result in no matings (Fig. 6), so that the actual probability is 0.125 , but the binomial approximation $\left(\left(\left(1-\frac{1}{r}\right)^{m}\right)^{f}\right)$ gives 0.0625 , a percentage error of $50 \%$. Whereas for six mating sites, we have an exact probability of 0.4861 and a binomial approximation of 0.4823 , a percentage error of less than $1 \%$ (Fig. 7).

We note, however, that this binomial approximation of the probability of mate encounter will perform poorly when used to estimate of the number of monogamous pairs because males that have been previously matched with a female are no longer available. For polygynous mating, the approximation performs well. To calculate the number of mated females where mating is polygynous, we use one minus $\left(1-\frac{1}{r}\right)^{m}$ to calculate the probability that there is a male at the site occupied by a given female, and assuming the events are independent, we can simply multiply this probability by the number of females to get an estimate of the number of mated females:

$E($ mated $)=\left[1-\left(1-\frac{1}{r}\right)^{m}\right] f$

\section{Appendix B}

In the main text, we used simulation to predict the probability of a given number of male:female pairs occurring

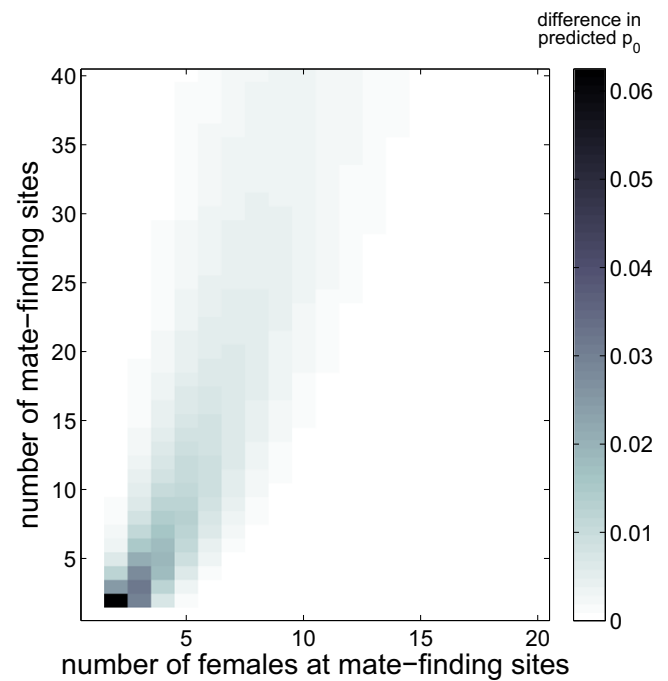

(1:1 sex ratio)

Fig. 7 The difference between the exact solution and the binomial approximation of the probability $p_{0}$ that there are no matched pairs, for a 1:1 sex ratio and a given number of females and landmarked sites across a given number of landmarked sites. A combinatorially exact solution to this problem does exist (Wendl 2005); however, it is computationally expensive. In the following, we provide an explanation of how to obtain this exact solution. We then describe our computational implementation of this solution, and show that the simulation results are a very good approximation of the exact solution.

For monogamy, the maximum number of mated pairs at a given site is given by the minimum number of males or females at that location. Each individual in the set of all females, $f$, and each individual in the set of all males, $m$, is randomly assignment to one of $r$ sites. Then, the total number of pairings is given as the sum over the number of individuals of either sex at each site: $k=$ $\sum_{l=1}^{l=r} \min \left(m_{l}, f_{l}\right)$.

Wendl (2005) provided an exact solution for the number of possible arrangements of two kinds of objects that gives a certain number of matches, $\mathrm{k}$ :

$$
\begin{array}{r}
H(m, f, k, r)=\sum_{i=1}^{m} \sum_{j=1}^{f} \sum_{\epsilon=1}^{\gamma(m, i)} \sum_{\eta=1}^{\gamma(f, j)} \theta(m, i, \epsilon) \theta(f, j, \eta) \\
\sum_{\kappa=1}^{k} \sum_{\phi=1}^{N(i, j, \kappa)} \delta_{k, \mu(m, f, i, j, \epsilon, \eta, \kappa, \phi)} E(i, j, \kappa, r)
\end{array}
$$

where $m$ is the number of males, $f$ is the number of females and $k$ is the number of matches between them over $r$ landmarked sites. This function is able to enumerate all cases where $0<k \leq \min (m, f)$. The special case where $k=0$ for any combination of $m, f$ and $r$ can be calculated using Stirling numbers of the second kind as described earlier in this paper and by Wendl (2003).

The first four summation terms in the equation for $H$ simply enumerate the number of ways all individuals in a population could be subdivided or partitioned given that we consider the set of all males and the set of all females independently. For example, four females could be partitioned in five possible ways: a single group of 4, (4), two groups of $2(2,2)$, a group of 1 and a group of $3(1$, $3)$, two groups of 1 and a group of $2(1,1,2)$, or four groups of $1(1,1,1,1)$. Of these arrangements, two result in the individuals being divided among two groups, or have order two: $(2,2)$ and $(1,3)$. We will wish to know the total number of subgroups in a partition when distributing individuals across sites (i.e. if individuals are divided into two groups and are distributed across three sites, then one site will be empty).

Partitions of arbitrarily large integers (number of individuals) are described by the Bell polynomials, and can be obtained using a generating function (Riordan 1968). Using 
notation similar to Riordan (1968) and Wendl (2005), the first four Bell polynomials are:

$$
\begin{aligned}
& Y_{1}=x^{1} g_{1} \\
& Y_{2}=x^{1} g_{2}+x^{2} g_{1}^{2} \\
& Y_{3}=x^{1} g_{3}+x^{2}\left(3 g_{1}{ }^{2} g_{2}\right)+x^{3} g_{1}{ }^{3} \\
& Y_{4}=x^{1} g_{4}+x^{2}\left(4 g_{1} g_{3}+3 g_{2}{ }^{2}\right)+x^{3}\left(6 g_{1}{ }^{2} g_{2}\right)+x^{4} g_{1}{ }^{4}
\end{aligned}
$$

The meaning of each term can be understood through the coefficients and subscripts. The equation $Y_{4}$ gives us the partitions for four individuals. We see there are five terms corresponding to the five possible arrangements. For each term, the subscript of each $g$ corresponds to the size of each partitioned element (i.e. the number of individuals in the group), while the exponent on $g$ gives the number of groups of that size in the partition. The first term $x^{1} g_{4}$ refers a group of four individuals and the implicit exponent of one on $g$ tells us there is only one group of this size in the partition. The term $g_{2} g_{1}{ }^{2}$ refers to a partition of one group of 2 and two groups of 1 . The exponent on $x$ for each term gives us the order of the partition. So $g_{2} g_{1}^{2} x^{3}$ tells us that one group of 2 and two groups of 1 has order 3 (the entire partition has three groups). Similarly, the $x^{2}$ for $4 g_{3} g_{1}$ and $3 g_{2}{ }^{2}$ indicates that both of these partitions are composed of two groups.

The first two summations in the equation for $H$ count through the different orders of the partition given $m$ males and $f$ females, where the highest order is when every individual is in its own group. In addition, we have to sum the number of ways we can have a partition of a given order, given as $\gamma$. For example, $\gamma(4,2)$ gives the number of partitions of order 2 in the 4th Bell polynomial, which we have just seen, equals 2 . The second pair of summation signs in the equation for $H$ counts through the number of possible partitions of a given order for the set of males and females.

Finally, the coefficient on each term of the Bell polynomial representing a possible partition tells us how many different ways there are to place identifiable individuals into these groups. So the compete term $4 g_{3} g_{1} x^{2}$ in our equation for $Y_{4}$ tells us there are four ways of partitioning four different individuals ( $a, b, c, d)$ into two groups, where one of these groups has three members, and the other has one member (i.e. [abc],[d]; [abd],[c]; [acd],[b] and [bcd],[a]). In the equation for $H$, the function $\theta$ refers to these coefficients of the Bell polynomials. For example, $\theta(4,2,1)$ refers to the first coefficient of partitions of order 2 of the 4th Bell polynomial, which we have just seen is equal to 4 . The coefficients gathered by each $\theta$ will multiply our solution out for all possible possible arrangements of identifiable individuals.

Once we have determined the number of ways our set of males and females can be partitioned for each arrangement, we also need to determine the many ways the subgroups in each set could be arranged relative to each other, and subsequently, how many of these arrangements result in a given number of matched pairs, $k$. First using the function N, we count through the number of ways a partition of males of order $i$ and a partition of females of order $j$ could be arranged such that $\kappa$ sites have both sexes:

$N(i, j, \kappa)=\frac{i ! j !}{(i-\kappa) !(j-\kappa) ! \kappa !}$

For example, if our partition of males is order 2 (two groups), and our partition of females is order 1 (one group), for $\kappa=1$, there are two possible arrangements $N=$ $\frac{2 ! 1 !}{(2-1) !(1-1 !) 1 !}$. For the case of four males and four females in a partition with order 2 and order 4, respectively, we can get both sexes at one site from eight different arrangements, given as $N=\frac{2 ! 4 !}{(2-1) !(4-1 !) 1 !}$. Initially, we can see that it is possible to have a male and female group at the same site in $i * j$ possible ways; however, the second time this occurs it can only happen in $(i-1) *(j-1)$ ways (since one site is already occupied), and so on. The result is divided by $\kappa$ ! to account for all possible orders of these events.

The total event space is determined by the number of distinct sites that these arrangements are distributed over:

$E(i, j, \kappa, r)=\prod_{\eta=0}^{i+j-\kappa-1}(r-\eta)$

Each group in the partition of females, and each group in the partition of males, can be found at one of $r$ sites. However, only some of these assignments result in $\kappa$ sites with both males and females. An event is when $\kappa$ groups of males and $\kappa$ groups of females have the same site value. The total possible number of distinct site values is $i+j$ (i.e. every group of males and every group of females at a different site). We are restricting our attention to the cases where there are $\kappa$ sites with both sexes, so the maximum possible number of occupied sites is therefore $i+j-\kappa$, since every site shared by the two sexes reduces the number of possible site values by one. Note that this counting value can have a different maximum value than the actual number of distinct sites, $r$.

To determine the possible number of events, from our given partition, we arbitrarily select a group of females. This group could take any one of $r$ site values. Since this site is now occupied, the next group of females then has $r-1$ site values that it could take and so on. For example, with a partition of males of order $1(i=1)$, and a partition of females with order $2(j=2)$, and three sites $(r=3)$, there are six $(E=6)$ possible ways to get one site $(\kappa=1)$ with both males and females.

For each way of arranging the partitions of males and females such that both sexes occur at $\kappa$ sites, there will be a number of matches $(k)$ between individual males and females. The number of matches at a given site where both 
Table 1 Actual and estimated runtimes to calculate the probability of more than five mated pairs for a given number of sites with both sexes $\kappa$

\begin{tabular}{llll}
\hline$\kappa$ & Actual runtime $(\mathrm{s})$ & $\kappa$ & Estimated runtime $(\mathrm{s})$ \\
\hline 1 & 2.4 & 6 & 198715.2 \\
2 & 23.0 & 7 & 1916532.5 \\
3 & 221.5 & 8 & 18484223.0 \\
4 & 2136.3 & 9 & 178273263.2 \\
5 & 20603.7 & 10 & 1719377454.8
\end{tabular}

sexes co-occur is given by the minimum of the two group sizes. The total number of matches is then the sum of this value over the number of sites with both sexes:

$\mu(m, f, i, j, \epsilon, \eta, \kappa, \phi)=\sum_{\psi=0}^{\kappa} \min \left(\alpha_{\psi}, \beta_{\psi}\right)$,

where $\alpha$ is the size of the group of males at site $\psi$ and $\beta$ is the size of the group of females at this site. After the total number of matches is calculated, this sum is evaluated against the global number of desired matches, $k$, by a Kronecker delta function (Kreyszig 1988). The $\delta$ function evaluates to 0 when $k$ is not equal to $\mu$, and to 1 when $k=\mu$.

In order to calculate the probability of a given number of matings, we will need to calculate the total possible number of matings. Consider our simple example again, for two sites and two males, there are $2^{2}=4$ ways of distributing the two males between the two sites. Given these four arrangements, we must then consider all possible ways of distributing our two females (also $2^{2}$ ). With each set of four distributions generated for males and females, we must then consider how many ways we might overlap each distribution. For each individual male distribution, we have four possible female distributions to choose from, so the total number of possible overlaps becomes the product of each number of possible distributions. In other words, the total number of match events through $k=0,1,2,, m$ can be calculated as: TotalEvents $=r^{m+f}$ With specific match event numbers generated for each $k$ value, and a total number of events known, we can generate a probability distribution for each $k$ value, i.e. the probability of $k$ matches occurring between $m$ males and $f$ females over $r$ sites:

$P_{(r, k)}=\frac{r^{m+f}-H(m, f, k, r)}{r^{m+f}}$

Computational implementation

We implemented a computational solution for $P_{r, k}$ in MATLAB (2013), and compared our simple simulation results to this exact solution.

This exact solution requires large computation time for large numbers of individuals. Therefore, to make our comparison to simulation results, we implemented solutions using only the first ten Bell polynomials, and so could construct a static reference matrix for the coefficients. The form of our reference matrix, shown here using only the first four Bell polynomials for illustration, is: $\left[\begin{array}{cccc}1 & 0 & 0 & 0 \\ 1 & 1 & 0 & 0 \\ 1 & 3 & 1 & 0 \\ 1 & (4,3) & 6 & 1\end{array}\right]$. The rows of the matrix correspond to the Bell polynomial of that degree. So, the fourth row corresponds to the fourth bell polynomial, $Y_{4}$. The columns correspond to the order of the partition, $j$. For example, the values $(4,3)$ in row 4 , column 2 mean there are $(4+3)=7$ total ways to partition four elements into two groups. Continuing along for each of the first ten Bell polynomials (Riordan 1968), we constructed a $10 \times 10$ matrix. Even so, the number
Table 2 Enumeration of $k>4$ matches for various numbers of sites, $r$

\begin{tabular}{lll}
\hline$r$ & Number of match events for $k>4$ & Total events $r^{m+f}$ \\
\hline 1 & 1 & 1 \\
2 & 1023794 & 1048576 \\
5 & 68604249041165 & 95367431640625 \\
10 & 41442721783252590592 & 1000000000000000000000 \\
15 & 86639700109715105120256 & 332525673007965060202496 \\
20 & 15464147417781939753451520 & 104857600000000000000000000 \\
35 & 221902578291667367473994268672 & 7609583501588058269346848309248 \\
50 & 75614213577185983339175293747200 & 9536743164062500289712932993892352 \\
\hline
\end{tabular}


Fig. 8 Agreement between simulated (over 1000000 replicates) and calculated probabilities of at least 5 matched females for different numbers of landmarked sites

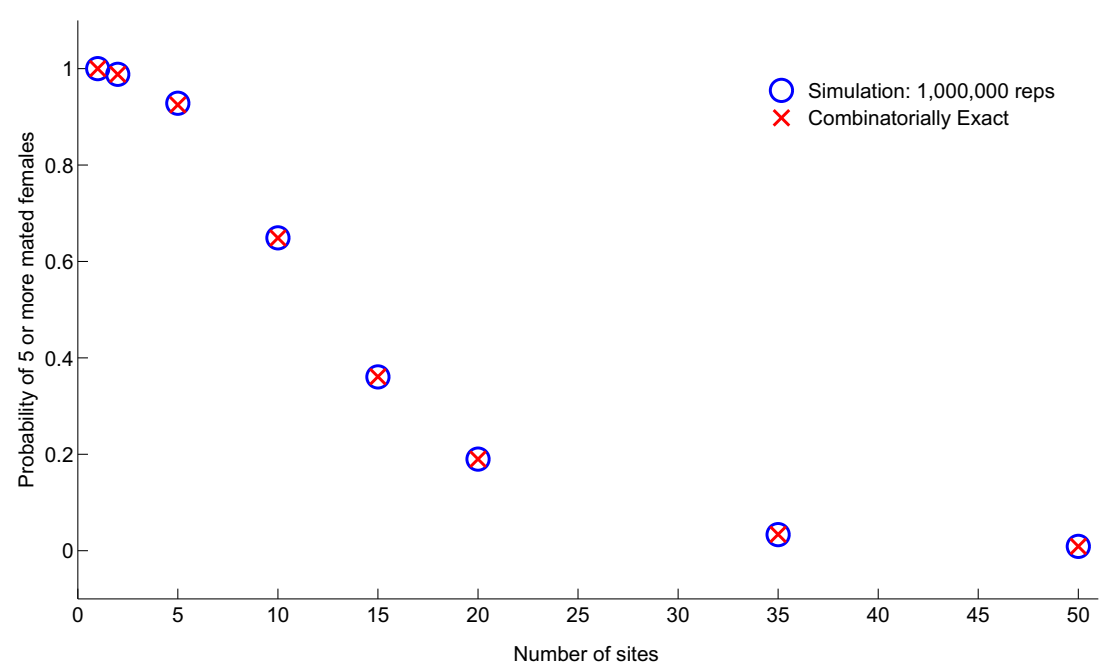

of loops required for each summation gives a function that must generate extremely large numbers of possible arrangements.

The production of all $N$ unique arrangements that produce $\kappa$ sites with both sexes is the most computationally expensive part of our implementation of the $H$ function, as $N$ becomes very large very quickly (e.g., $N(10,10,7)=$ $31,752,000)$. The recursive generating function written to compute these arrangements outputs a matrix where each row corresponds to the male and female groups at the same site. We will refer to this matrix as the N-matrix. For all 31,752,000 possible arrangements, we would get an $\mathrm{N}$-matrix with 31,752,000 rows and 14 columns. All N rows are passed one at a time $(\phi=1: N)$ to the accumulating function, $\mu$, to calculate the number of matched male-female pairs.

For this implementation, we calculated the runtime for up to five sites with both sexes. The bottleneck of generating a matrix with all possible $\mathrm{N}$-value pairs gives us a function with approximately exponential runtime relative to the number of sites with both sexes $(\kappa)$. Projection from these times for six or more shared sites suggest unreasonably long runtimes (Table 1 ). We conclude that simulation is a more time-efficient solution for this problem.

However, simulation may introduce errors. To evaluate the accuracy of simulated solutions, we ran the exact solution for ten individuals of each sex $m=f=10$, and four pairings, $k=1,2,3,4$ across various numbers of sites, $r=1,2,5,10,15,20,35,50$ (Table 2). Taking the difference between the sum of all enumerations for $k=$ $0,1,2,3,4$ and the total number of possible match events, $r^{m+f}$, gives us the number of possible ways to distribute individuals such that five or more pairings occur. The quotient of this $k>4$ number and the total number of possible match events gives us a probability that five or more matings will occur.

Simulations with sufficient replicates yield estimated probabilities that are indistinguishable from probabilities generated using the exact solution (Fig. 8). Therefore, while enumeration of all possible connectors is possible for a small number of individuals, in the main text, we elected to use simulated values. The simulated solutions accurately reflect the exact solution over many $m, f, k$, and $r$ values, and require significantly shorter runtimes.

\section{References}

Boswell M, Patil G (1970) Chance mechanisms generating the negative binomial distribution. In: Patil G (ed) Random counts in models and structures. Pennsylvania State University Press, University Park, London, pp 1-22

Braby M, Thistleton B, Neal M (2014) Host plants, biology and distribution of Acraea terpsicore (Linnaeus, 1758) (Lepidoptera: Nymphalidae): a new butterfly for northern Australia with potential invasive status. Austral Entomol 53:288-297

Costa-Pierce BA (1992) Review of the spawning requirements and feeding ecology of silver carp (Hypophthalmichthys molitrix) and reevaluation of its use in fisheries and aquaculture. Rev Aquat Sci 6:257-273

Cuddington K, Currie WJS, Koops MA (2014) Could an Asian carp population establish in the Great Lakes from a small introduction? Biol Inv 16:903-917

Cudmore B, Mandrak N, Dettmers J, Chapman D, Kolar C (2012) Binational ecological risk assessment of Bigheaded carps (Hypophthalmichthys spp.) for the Great Lakes basin. DFO Can Sci Advis Sec Res Doc. 2011/114

Daugherty DJ, Sutton TM, Elliott RF (2008) Potential for reintroduction of lake sturgeon in five northern Lake Michigan tributaries: a habitat suitability perspective. Aquat Conserv 18: 692-702 
Dennis M (1989) Allee effects: population growth, critical density and the chance of extinction. Nat Res Model 3:481-538

Deredec A, Courchamp F (2007) Importance of the Allee effect for reintroductions. Ecoscience 14:440-451

Drake J, Lodge D (2006) Allee effects, propagule pressure and the probability of establishment: risk analysis for biological invasions. Biol Inv 8:365-375

Dubois F, Cezilly F, Pagel M (1998) Mate fidelity and coloniality in waterbirds: a comparative analysis. Oecologia 116: 433-440

Feller W (1957) An introduction to probability theory and its application, vol 1, 2nd edn. Wiley, New York, p 461

Frank KT, Brickman D (2000) Allee effects and compensatory population dynamics within a stock complex. Can J Fish Aquat Sci 57:5130-517

Gascoigne J, Berec L, Gregaroy S, Courchamp F (2009) Dangerously few liaisons: a review of mate-finding Allee effects. Pop Ecol $51: 355-372$

Hopper K, Roush R (1993) Mate finding, dispersal, number released, and the success of biological-control introductions. Ecol Entomol $18: 321-331$

Kahneman D, Slovic P, Tversky A (eds) (1982) Judgement under uncertainty: Heuristics and biases. Cambridge University Press, Cambridge

Knipling E (1955) Possibilities of insect control or eradication through the use of sexually sterile males. J Econ Entomol 48: 459-462

Kocovsky P, Chapman D, McKenna J (2012) Thermal and hydrologic suitability of Lake Erie and its major tributaries for spawning of Asian carps. J Great Lakes Res 38:159-166

Kolar C, Chapman D, Courtenay W, Housel C, Williams JD, Jennings, D (2005) Asian carps of the genus Hypophthalmichthys (Pisces, Cyrprinidae) - a biological synopsis and environmental risk assessment. Report to the U.S. Fish and Wildlife Service. U.S. Geological Survey, LaCrosse, Wisconsin

Kramer A, Sarnelle O, Knapp R (2008) Allee effect limits colonisation success of sexually reproducing zooplankton. Ecology 89:2760 2769

Kramer A, Dennis B, Liebhold A, Drake J (2009) The evidence for Allee effects. Pop Ecol 51:341-354

Kreyszig E (1988) Advanced engineering mathematics, 6th edn. Wiley, New York

MATLAB (2013) The MathWorks, Inc., Natick, Massachusetts

Mills D (1989) Ecology and management of Atlantic Salmon. Chapman and Hall, New York

Nakata T (2008) Collision probability for an occupancy problem. Stat Prob Letts 78:1929-1932

Painter K (2014) Multiscale models for movement in oriented environments and their application to hilltopping in butterflies. Theor Ecol 7:530-75

Pe'er G, Saltz D, Thulke H, Motro U (2004) Response to topography in a hilltopping butterfly and implications for modelling nonrandom dispersal. Anim Behav 68:825-839

Pe'er G, Heinz SK, Frank K (2006) Connectivity in heterogeneous landscapes: analyzing the effect of topography. Land Ecol 21:4761

Purchase C, Hutchings JA (2008) A temporally stable spatial pattern in the spawner density of a freshwater fish: evidence for an ideal despotic distribution. Can J Fish Aquat Sci 65:382-388
Rakes PL, Shute JR, Shute PW (1999) Reproductive behavior, captive breeding, and restoration ecology of endangered fishes. Env Biol Fish 55:31-42

Reed JM, Dobson A (1993) Behavioural constraints and conservation biology: conspecific attraction and recruitment. Trends Ecol Evo $8: 253-256$

Riordan J (1968) Combinatorial identities. Wiley, New York

Rowe S, Hutchings JA (2003) Mating systems and the conservation of commercially exploited marine fish. Trends Ecol Evo 18:567-572

Rutowski R (1991) The evolution of male mate-locating behavior in butterflies. Am Nat 138:1121-1139

Ruxton G, Bailey D (2005) Combining motility and bioluminescent signalling aids mate finding in deep-sea fish: a simulation study. Mar Ecol Prog Ser 293:253-262

Schnabel Z (1938) The estimation of the total fish population of a lake. American Mathematical Monthly 45:348-352

Scott J (1970) Hilltopping as a mechanisms to aid the survival of low density species. J Res Lepidoptera 7:191-204

Shields O (1967) Hilltopping: an ecological study of summit congregation behaviour of butterflies on a southern California Hill. J Res Lepidoptera 6:69-178

Skevington J (2008) Hilltopping. In: Capinera J (ed) Encyclopedia of entomology, vol 2. Springer, pp 1799-1807

Skjraasen J, Meager J, Karlsen O, Hutchings JA, Ferno A (2011) Extreme spawning-site fidelity in atlantic cod. ICES J Mar Sci 68:1472-1477

Soderlund C, Humphray A, Dunham A et al. (2000) Contigs built with fingerprints, markers, and FPC V4.7. Genome Res 10:17721787

Taylor CM, Hastings A (2005) Allee effects in biological invasions. Ecol Letts 8:895-908

Thornhill R, Alcock J (1983) The evolution of insect mating systems. Harvard University Press, Cambridge

Twohey M, Henirich J, Seelye J, Fredricks K, Bergestedt R, Kaye C, Scholefield R, McDonald R, Christie G (2003) The sterile-malerelease technique in Great Lakes sea lamprey management. J Great Lakes Res 29:410-423

Veit R, Lewis M (1996) Dispersal, population growth and the Allee effect: dynamics of the house finch invasion of eastern North America. Am Nat 148:255-274

Walter SD (1980) Large sample formulae for the expected number of matches in a category matched design. Biometrics 36 : 285-291

Wendl M (2003) Collision probability between sets of random variables. Stats Prob Letts 64:249-254

Wendl M (2005) Probabilistic assessment of clone overlaps in DNA fingerprint mapping via a priori models. J Comp Biol 12: 283-297

Wickman P (1988) Dynamics of mate-searching behaviour in a hilltopping butterfly, Lasiommata megera (L.): the effect of weather and male density. Zoo J Linn Soc 93: 357-377

Wickman P (1994) The location of landmark leks in the small heath butterfly, Coenonympha pamphilus: evidence against the hot-spot model. Behav Ecol 6:39-45

Young K, Genner M, Joyce D, Haesler M (2009) Hotshots, hot spots and female preference: exploring lek formation models with a bower-building cichlid fish. Behav Ecol 20: $609-615$ 